\title{
SolAgua, a non static compound parabolic concentrator (CPC) for residential and service buildings
}

\author{
P. Gata Amaral a, b, E. Ribeiro ${ }^{\text {a }}$, R. Brites ${ }^{\text {a }}$, F. Gaspar ${ }^{\text {a }}$ \\ a School of Technology and Management, Polytechnic Institute of Leiria (ESTG-IPL), \\ Morro do Leno - Alto do Vieiro, 2411-901 Leiria, P. O. Box 4163, Portugal \\ phone: +351 244820 300, fax: +351 244820 310, e-mail: pgata@estg.ipleiria.pt, eliseu@estg.ipleiri.pt \\ ${ }^{\mathrm{b}}$ Institute for Systems and Computers Engineering of Coimbra (INESC Coimbra) \\ Rua Antero de Quental, 199, 3000-033 Coimbra, Portugal \\ phone: +351 239851 040, fax: +351239824 692
}

\begin{abstract}
This paper presents a brief description of the SolAgua project, which aims at the design, development and conception of a non plan solar collector that permits the maximum capture of Solar Energy. This developed solar collector was based on a format known as Compound Parabolic Concentrator (CPC), which has already been sufficiently used at a world-wide range. The system developed during this project presents results, which can be considered sufficiently satisfactory, being able of heating the water from $45^{\circ} \mathrm{C}$ to $60^{\circ} \mathrm{C}$. These results can be achieved to a great extent owing to the movement of rotation of the SolAgua collector provided by its innovative automatic support. This movement enables it to follow the land movement in relation to the Sun.
\end{abstract}

Key-words: Solar thermal energy; Process monitoring; Compound parabolic concentrators, Sun orientation system.

\section{Introduction}

Portugal is very dependant on the external energy supplies. This fact makes the country extremely vulnerable not only in what concerns the ensuring of the supplies but also national supporting of the energy costs. Besides this, Portugal is also one of the countries that have signed the Quioto protocol, which means a commitment with the achievement of the environmental goals proposed.

Nevertheless, Portugal's climatic conditions are extremely favourable to the use of Solar Energy, since there are in average mare than 2.000 hours of sunlight a year.

The SolAgua project was born with the purpose of improving and evaluating the existing potential in the use of the Solar Energy for the heating of water in residential buildings and services. The potential of these systems are very high in countries like Portugal with a high number of clear Sun light hours a year.

The main purpose and the big challenge of the project were to improve the already high performance of the CPC collectors. The functioning of the new solar collector was therefore designed, built and monitored.
The advantages of the new solar collector are its great versatility, the low costs and higher energy efficiency. This article summarises the concept, the design and the mechanical structure of the SolAgua collector.

\section{Conception and sizing of the system}

\section{A. SolAgua Collector Specification}

The SolAgua collector made use of the topology CPC (vd. Fig.1). This format is currently world-wide used in a largescale level, since this type of solar collector ensures a better performance in comparison with the conventional plain collectors.

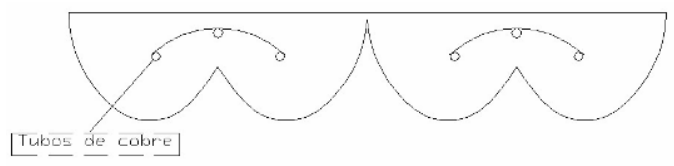

Fig. 1 - SolAgua CPC collector, plan of the prototype.

The materials used in the construction were carefully chosen in order to ensure a high resistance to high temperatures and to prevent incompatibilities of the materials.

\section{B. Orientation System}

In order to improve efficiency the SolAgua integrates an active system in order to move the collector during the day. There was the necessity of creating an orientation system that allowed the collector to move both horizontally and vertically. It was therefore designed and constructed a support which enabled a rotation of the SolAgua collector with a horizontal scanning angle of $200^{\circ}$ and that allowed an inclination with an angle of vertical scanning of $40^{\circ}$. The two levels of the movement that this support enables are made by the means of two motor reducers with a power of $120 \mathrm{~W}$ each. The orientation system is completely automatic and enables the SolAgua collector to follow the land movement in relation to the sun. Figure 2 shows the SolAgua Orientation System. 


\section{C). Hydraulic Installation}

The development of the SolAgua system also included the design and the installation of the intrinsic hydraulic installation (Fig.3). The installation is totally automated controlled in order to enable the normal functioning of the system, the storage and the distribution of hot water and for evaluating the collector's performance.

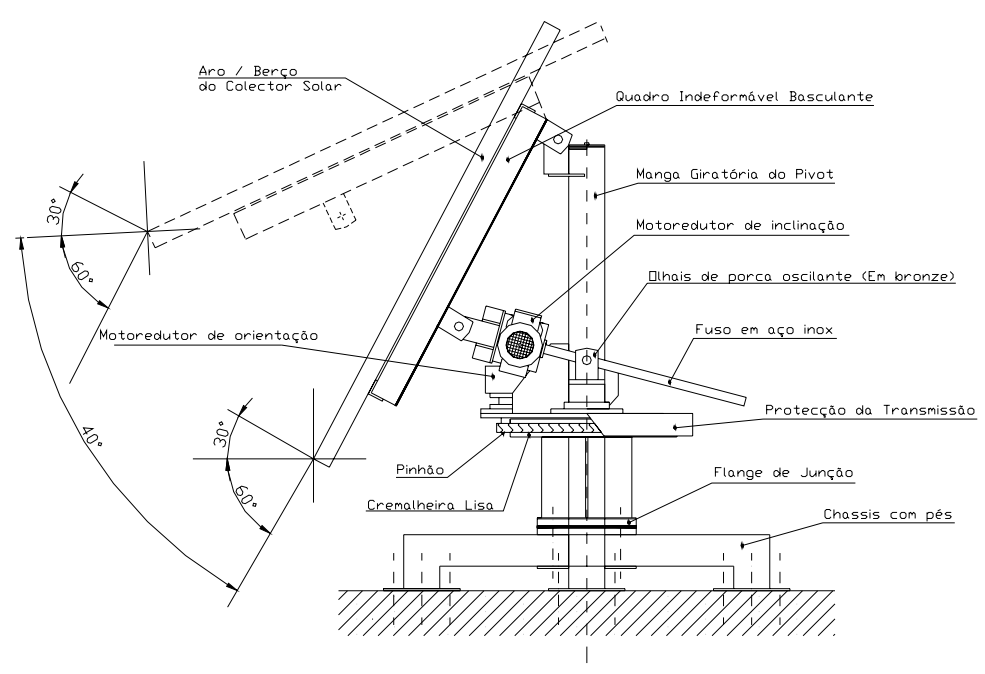

Fig. 2 - Design of the orientation system.

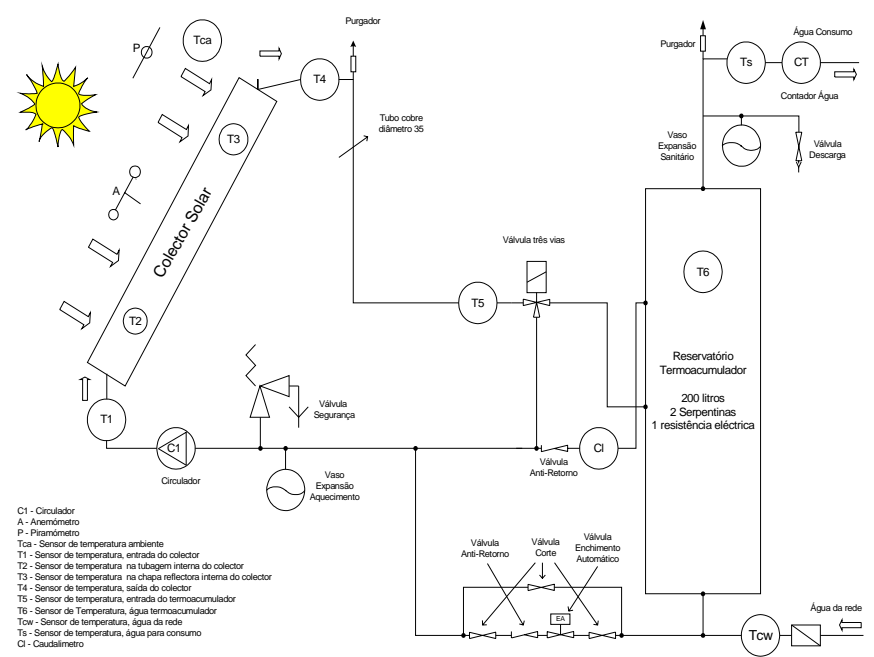

Fig. 3 - Hydraulic installation diagram.

\section{Monitoring an Control System}

The monitoring and control system based on a programmable logical controller (PLC), on a data logger and on a sensorial net enables to monitor and to store data on the following variables:

- Solar radiation, global and diffuse;

- Insulation;

- Wind speed;

- Ambient temperature;

- Temperature of the water to heat in the entrance of the collector;

- Temperature in the internal pipe of the collector;

- Temperature in the internal reflector base of the collector;
- Temperature of water at the exit of the collector;

- Temperature of the hot water at the entrance of the hot water tank;

- Temperature of the water inside the hot water tank;

- Temperature of the hot water for consumption at the exit of the tank;

- Temperature of the water of the net distribution;

- $\quad$ Temperature inside the collector;

- Temperature in the left reflector;

- Temperature in the right reflector;

- Temperature in the left absorber;

- Temperature in the right absorber.

These variables are collected with different sensors and stored in the data logger. 
The control system based on a PLC uses some of those variables, for the automatic control of:

- $\quad$ The pump of the primary circuit;

- The three ways valve used in the hydraulic installation;

- The orientation system.

\section{SolAgua final system}

The Figure 4 shows the final aspect of SolAgua system after the construction and the installation of all its hydraulic, mechanic and electric components.

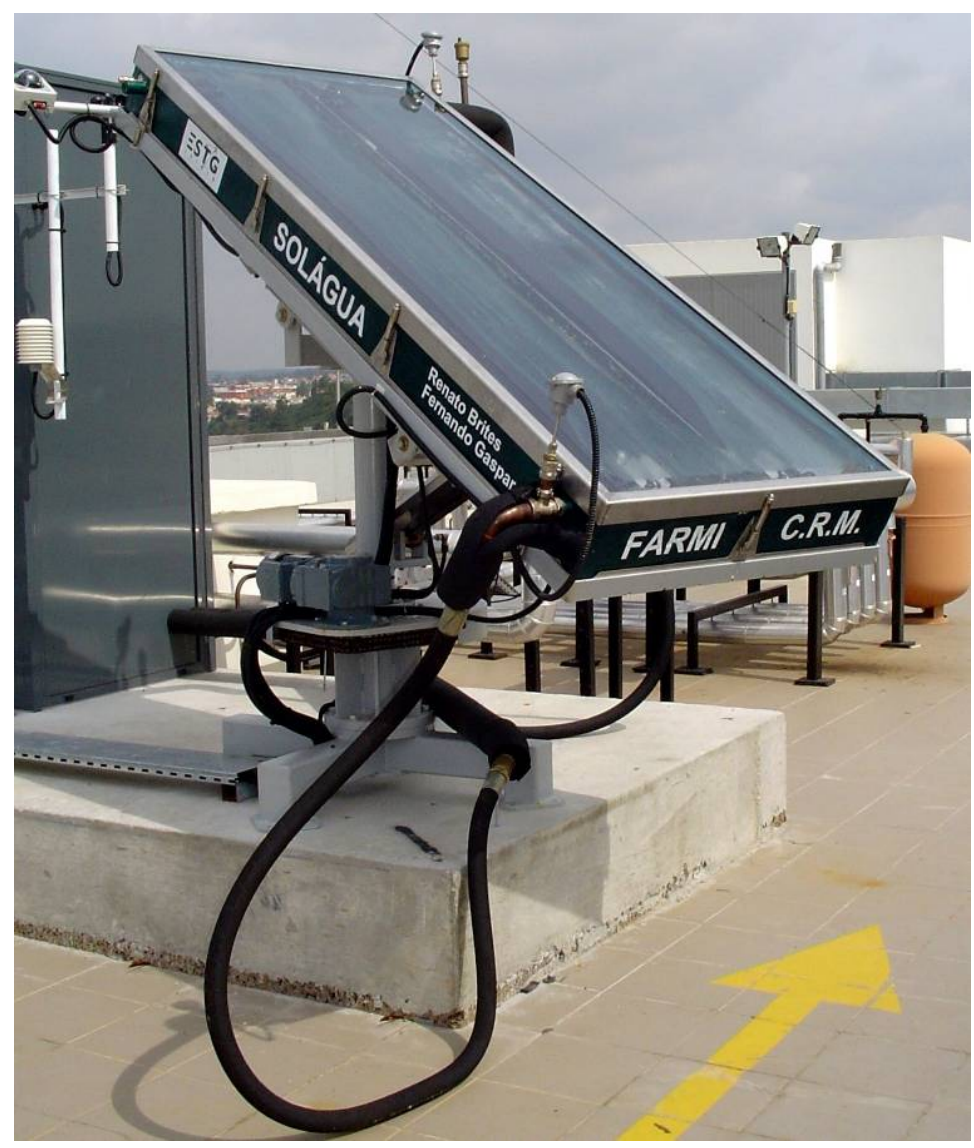

Fig. 4 - SolAgua CPC collector.

\section{Experimental results}

Several assays were carried through after the development and installation of the system in order to evaluate its viability and efficiency. The evaluation of the system's performance was carried through under different circumstances e.g. the following ones:

- Assay 1 - Manual mode system without hot water consumption and clear sky;

- $\quad$ Assay 2 - Automatic mode system, positioned at midday, without movement of the collector, without hot water consumption and clear sky;
- Assay 3 - Automatic mode system, with automatic following of the land movement in relation to the sun; without hot water consumption and clear sky;

- Assay 4-Automatic mode system, with automatic following of the land movement in relation to the sun; with hot water consumption and clear sky;

- $\quad$ Assay 5 - Automatic mode system, with automatic following of the land movement in relation to the sun; without hot water consumption and cloudy sky; 
The results of each assay are presented in Figures 5 to 9 .

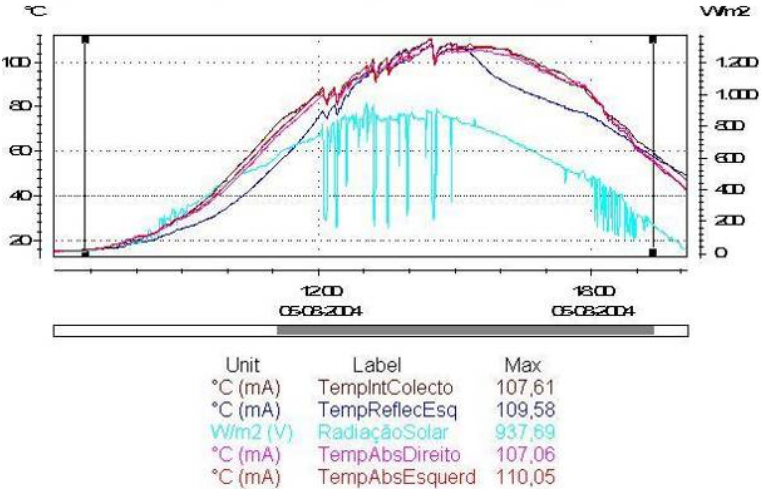

(a)

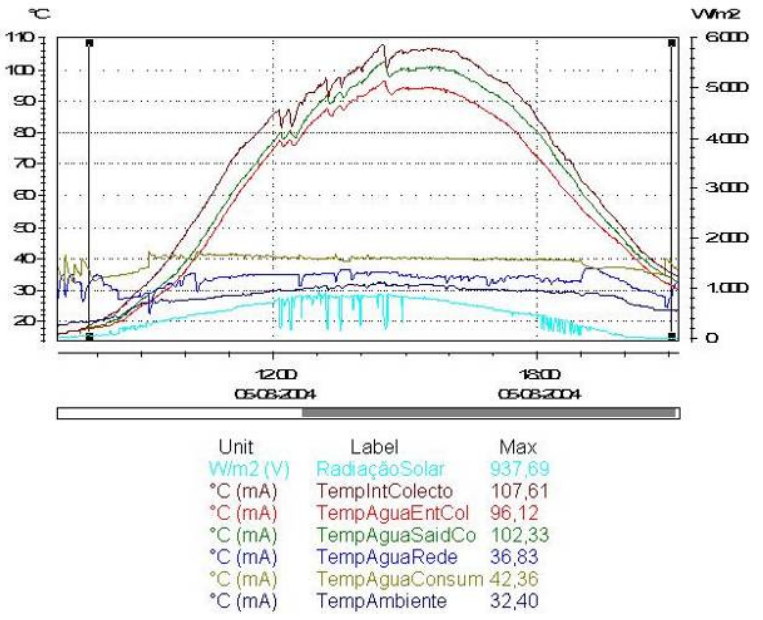

(b)

Fig. 5 - Assay 1 (a) Temperature in the SolAgua collector

(b) Water temperature.

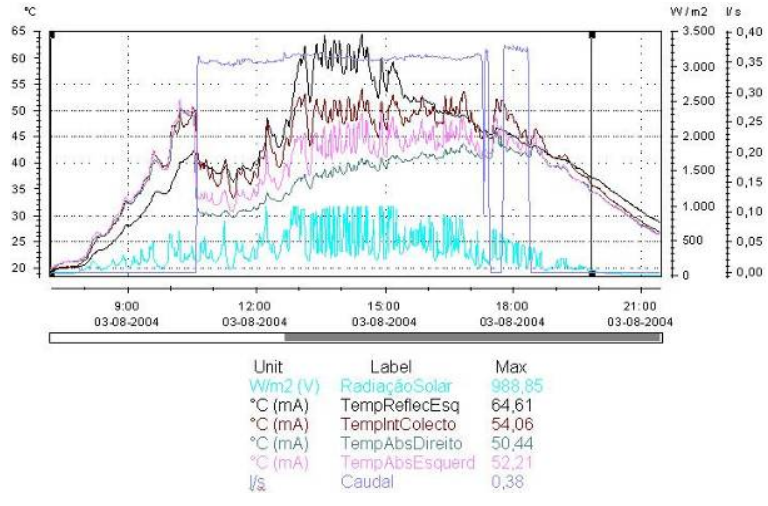

(a)

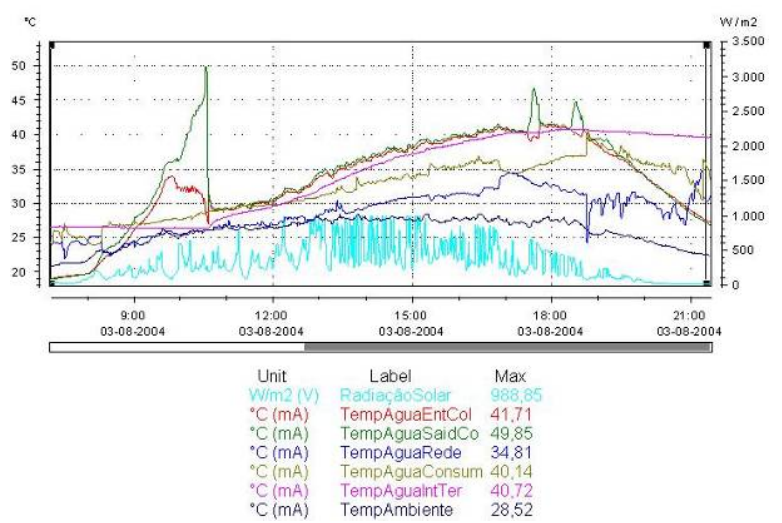

(b)

Fig. 6 - Assay 2 (a) Temperature in the SolAgua collector

(b) Water temperature.

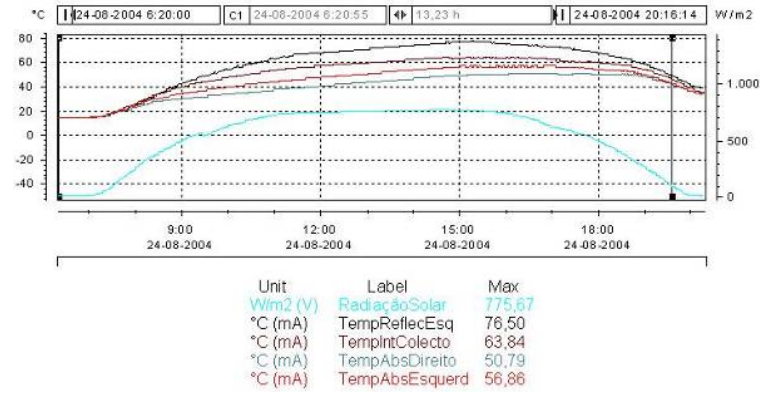

(a)

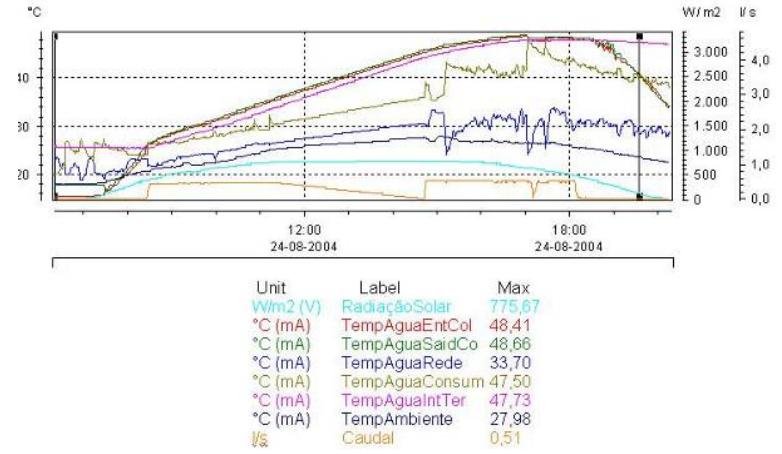

(b)
Fig. 7 - Assay 3 (a) Temperature in the SolAgua collector 


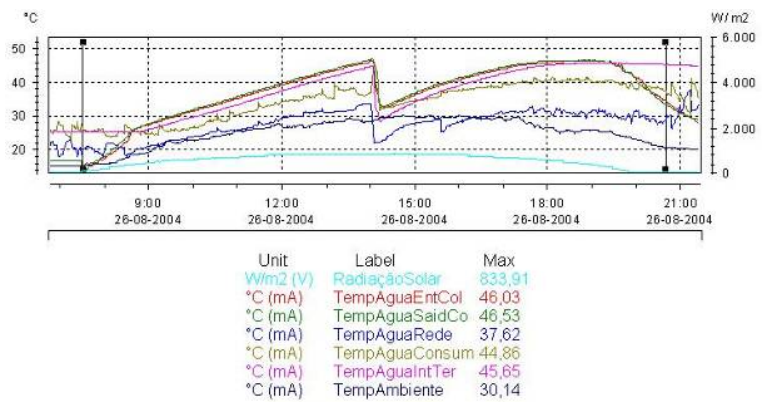

(b)

Fig. 8 - Assay 4 (b) Water temperature.

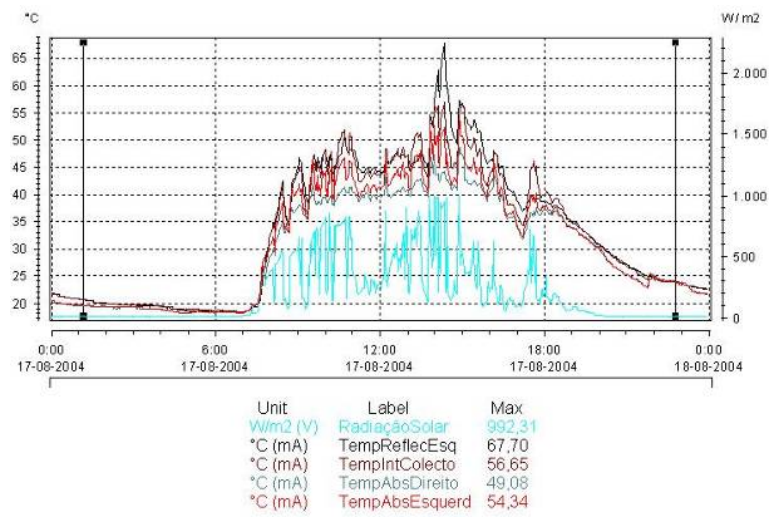

(a)

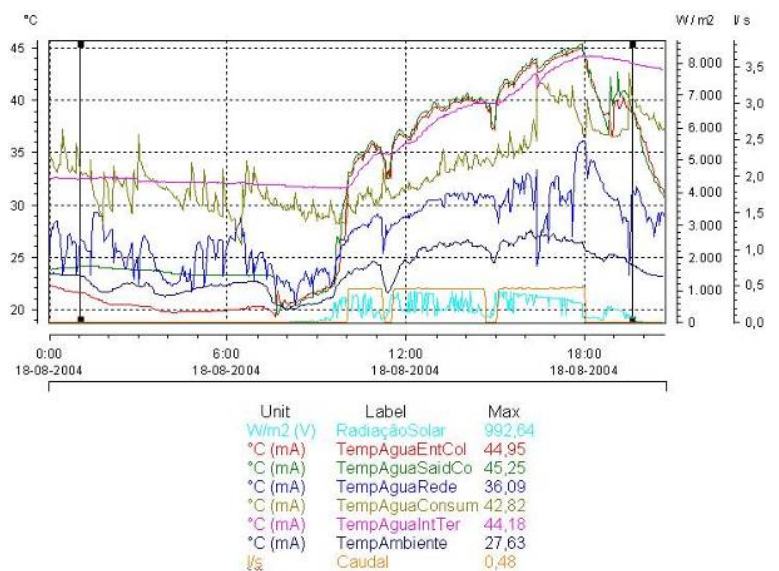

(b)

Fig. 9 - Assay 5 (a) Temperature in the SolAgua collector

(b) Water temperature.

\section{Useful energy and efficiency of the solar collector calculation}

The method used to calculate the useful energy was based on the equation of Bliss:

$$
\mathrm{Pu}=\mathrm{F}^{\prime} \times \mathrm{A} \times[\mathrm{Ig} \times \tau \times \alpha-\mathrm{UL} \times(\mathrm{tf}-\mathrm{ta})]
$$

Where:

Pu - useful energy;

F' - corrector factor;

A - collector surface;

Ig - incident global radiation on the collector;

$\tau$ - transmittance of the transparent covering;

$\alpha$ - absorbance of the absorber plate;

UL - global coefficient of losses

tf - average temperature of the fluid;

ta - ambient temperature;
The efficiency of the solar collector is given by:

$$
\eta=\mathrm{Pu} /(\mathrm{A} \times \mathrm{Ig})
$$

Using de values of Assay 3 it is possible to calculate the $\mathrm{Pu}$ and $\eta$ :

$$
\begin{aligned}
& \mathrm{P}_{\mathrm{u}}=629,94\left(\mathrm{~W} /{ }^{\circ} \mathrm{C} \cdot \mathrm{m}^{2}\right) . \\
& \eta=66,32 \% ;
\end{aligned}
$$

\section{Some conclusions}

Considering the obtained results and the calculated value of the collector's efficiency, we may conclude that the collector has a very good performance and that it can be implemented in heating of water both for residential and services buildings. 
The new orientation system used in the project seems to be an advantage since it enables the collector to have a more regular performance during the day. It also enables it to maximize its functioning at the beginning of the morning and at the end of the afternoon. In conclusion, this assay shows that the SolAgua collector is able to catch a higher quantity of solar radiation during the day. This new system also makes it possible to optimize the functioning of the collector on cloudy days. This fact means the possibility of successfully implementing the collector in other European countries with fewer sunlight hours (e.g. in the northern or central Europe).

We've also determined the net present value (NPV) of the project and the internal rate of return (IRR), and we've verified that the project presented a positive NPV value and a IRR which was superior to the interest rates of the market, more or less 3\% in Portugal, for supporting projects related to renewable energies.

\section{Acknowledgement}

The authors would like to thank the facilities and financial support provided by the School of Technology and Management of the Polytechnic Institute of Leiria.

\section{References}

[1] Rabl, Ari, Active Solar Collectors and Their Applications, Oxford University Press, Oxford (1996), pp. 147-156.

[2] Lebeña, Eduardo Perez (SPES) e Costa, Jorge Cruz (INETI), Manual de Instaladores de Equipamentos Solares Térmicos, Sociedade Portuguesa de Energia Solar (SPES), Lisboa.

[3] Collares Pereira, Manuel, Energias Renováveis, A Opção Inadiável, $1^{a}$ edição, INETI, Lisboa (1998).

[3] ISO 9459, Outubro de 2003. 